\title{
On Obtaining the Young Modulus from Numerical Analysis of Composite Material Constituent
}

\begin{abstract}
SORIN DRAGHICI ${ }^{1}$, HORIA ALEXANDRU PETRESCU ${ }^{1 *}$, ANTON HADAR ${ }^{1,2}$
${ }^{1}$ University Politehnica of Bucharest, Department of Strength of Materials, 313 Splaiul Independentei, 060032, Bucharest, Romania ${ }^{2}$ Academy of Romanian Scientists, 54 Splaiul Independentei, 050094, Bucharest, Romania

Importance and use of composite materials are no longer a subject that should be emphasized. They offer a successful replacement for classical materials in most areas of engineering, conferring similar elasticmechanical properties to metal or non-metal alloys with several advantages such as reduced mass, chemical resistance etc. Considering this, knowledge of the elastic-mechanical characteristics is of utmostimportance. The present article aims to create a finite element model that can predict the longitudinal elastic modulus of a double-layered composite material based on the elastic characteristics of its constituents. For this, the elastic characteristics of the constituents were determined, then used in the finite element analysis thus obtaining the Young's modulus for the numerical composite material. Also, the longitudinal elastic modulus of the resultant composite was determined experimentally. The results of the finite element model were compared with experimental values.
\end{abstract}

Keywords: composites, fiberglass, Young's modulus, FEM

In recent years, detailed analyses and experiments regarding the behavioural pattern of composite materials took a turn for faster and cheaper mechanical characterization. Previous methods consisted only of expensive and time-consuming experimental methods for obtaining the elastic characteristics of composite materials. A numerical approach, such as finite element modelling (FEM), offers faster, cheaper and more detailed results for an infinite number of composite materials configurations.

A series of relatively simple mechanical tests were used to evaluate the properties of the material considered. The results are used in engineering design and as a basis for comparing and selecting materials. This approach is presented in paper [1], inferring proper results for tree point bending numerical simulation compared to experimental sets. Dian-sen Li et al., present a parameterized finite element model [2] established for 3-Dimensional fivedirectional rectangular braided composite. A prediction of the effective elastic properties and the meso-scale mechanical response been presented to verify the validation of the FEM.

Stiffness degradation and failure morphologies obtained from the FEM results were presented in paper [3]. The stress distributions, stress hysteresis and failures of fibre tows and resins at different parts of the 3-D braided composite material have been collected from the FEM calculations to analyse the fatigue failure mechanisms.

The strategy presented in this paper is conceived with the aim of avoiding some of the numerical problems commonly encountered using most of the methodologies proposed in the literature for the mesoscale modelling of ceramic woven composites [4].

A good foundation for a successful FEM analysis consists in proper boundary conditions. A detailed approach is presented in the work [5] of S. Jaques et al. by creating a method for the construction of meso-scale FE models of textile reinforced composites using periodic boundary conditions on multiple part meshes. These practices can be applied even on more complex structures such as sandwich panels as presented in paper [6].
In analogy with the experimental methods presented in the work [7] of Siddiqui, M. Z., Tariq, F. etal., for composite laminates, the Young's modulus ( $E$ ), is termed as a quantity for the chord modulus as presented in figure 1 , and is calculated as:

$$
E=\frac{{ }^{p_{u}-P_{l}}}{A \times\left(\varepsilon_{u}-\varepsilon_{l}\right)}
$$

where:

$E$ is the chord modulus as defined in figure 1;

$P_{-}$is the tensile load at the upper strain limit;

$P_{1}$ - is the tensile load at the lower strain limit;

$\varepsilon$ is the upper strain limit;

$\varepsilon_{i}$ is the lower strain limit;

$A$ is the transversal area of the specimen.

In general, the tensile test is performed to evaluate the elastic constants, the strength, the ductility, and the strain hardening of the materials. The modulus of elasticity, $E$, is determined as a measure of rigidity, the yield strength limit, $\sigma_{\mathrm{y}^{\prime}}$ and the tensile strength, $\sigma_{\mathrm{t}}[8]$.

Detailed information regarding the tensile tests on composite materials is presented in paper [9], approaching the experimentata macro scale of the composite material. A micro scale inquiry is presented in the work [10] of $L i$, . ., J iao etal., not the subject of current paper but a forecast of

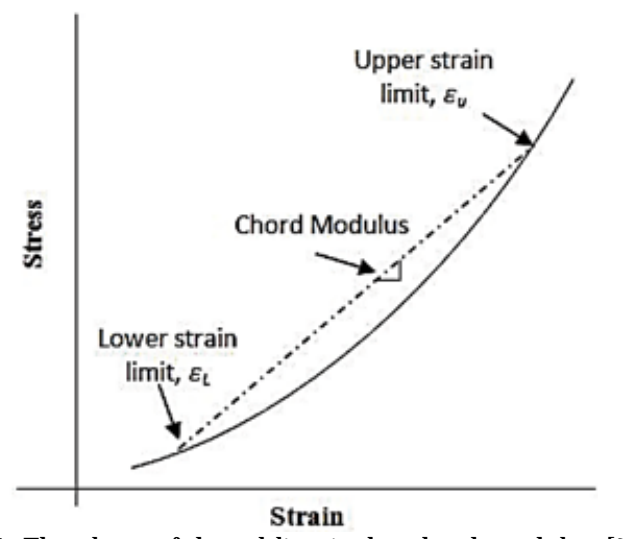

Fig.1. The slope of dotted line is the chord modulus [7] 
further investigations. A closer approach to our current interest is presented in paper [11].

Papers from Kabelka [12], Woo et al. [13], Sankar and Marrey [14] presented solutions for 2D analyses of plain weave composites using the assumption of plain-strain state, but these models are not suitable for correctly modelling textile composites.

Considering all of the above, the authors could establish a proper protocol for creating a 3D model and obtaining the Young's modulus for a fibreglass reinforced polymer composite material. At first, the experimental data, such as the strain-stress curves and Young's modulus, were obtained the constituents (fibreglass yarn and polymer matrix) and for a double layered composite. Constituent data was used for creating a finite element model and the composite data was used for validation of the numerical model.

\section{Experimental part}

Mechanical tests to determine strain-stress were performed on the Instron 8801 Universal Test Machine, thus evaluating five double layered samples for the longitudinal direction as shown in figure 2 [8].

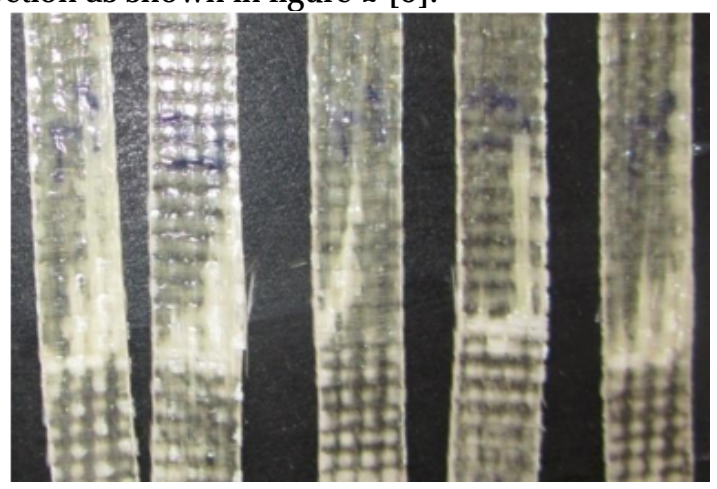

Fig. 2. Two-layer composite specimens for longitudinal direction

The results obtained were presented in figure 3 and in table 1. All calculations were machine performed such no alteration could be possible.

For the analytical calculation necessary to determine the Young's modulus for the constituents. A total of five polyester resin (fig. 4.a) and five fiberglass yarns samples (fig. 4.c) were prepared. All tests were performed on the Instron 8872 universal testing machine, using a strain gauge as depicted in figures $4 . b$ and 4.d. Four aluminium plates, two on each end of the fibreglass yarn, with a $50 \mathrm{~mm}$ separation were used in order to attach the strain gauge.

$$
\mathrm{sp}
$$

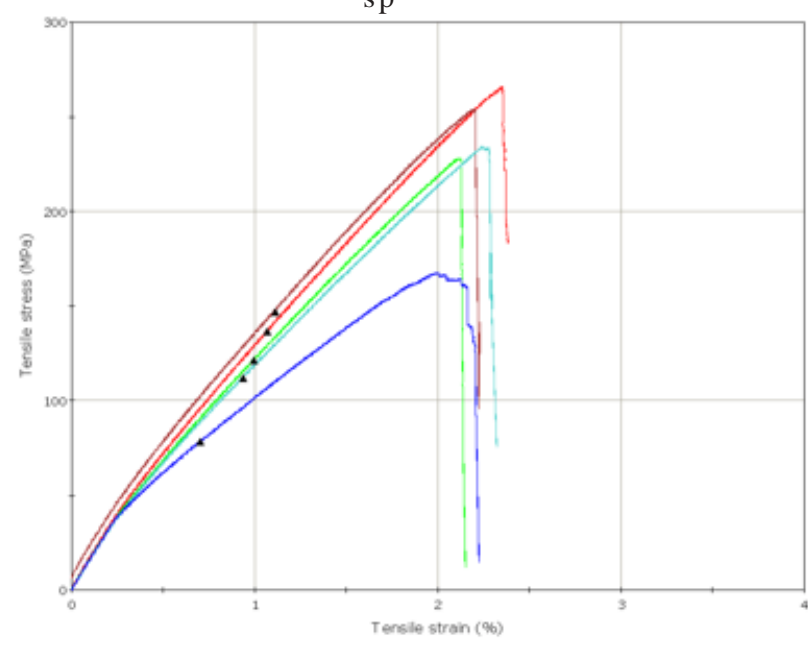

Fig. 3. Characteristic curves obtained for fibreglass-polyester resin with two layers in the longitudinal direction

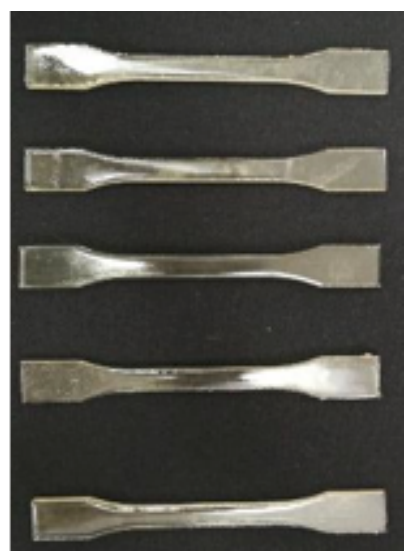

a. Resin specimens

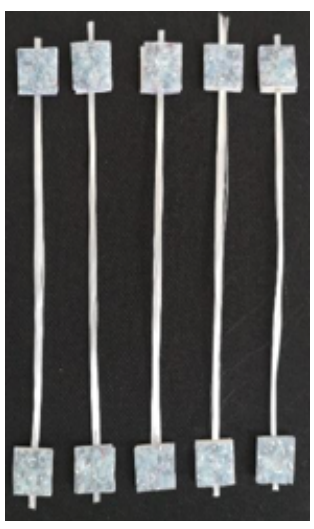

c. Fiberglass specimens

Fig. 4. The specimens and setup for experimental determination on constituents

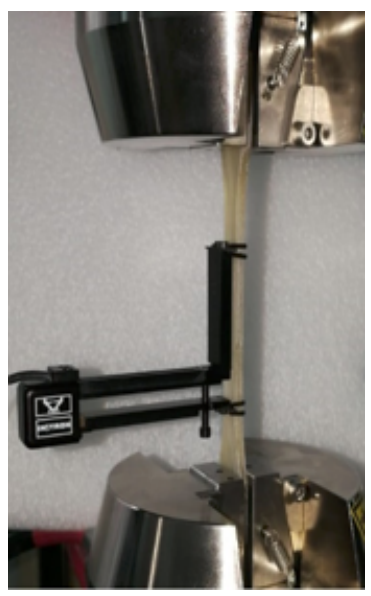

b. Experimental setup for resin

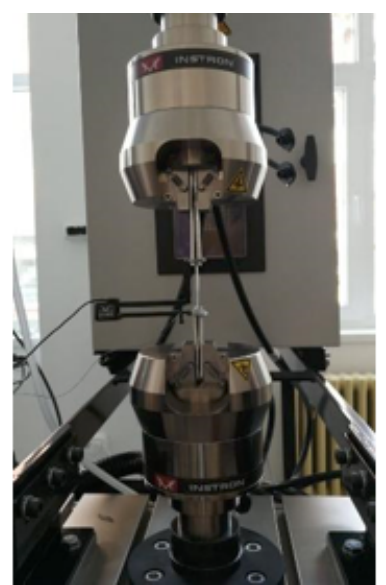

d. Experimental setup for

fiberglass
Figure 5 presents the strain-stress curve for the fibreglass yarn. The prestress difference between filaments, caused by the used aluminium brackets, generates small differences in tensile strength and strain origin for each specimen. These differences, as is shown in table 2, do not generate a large interval of data for the mean Young's modulus or tensile strength.

Tensile test results for the polyester resin presented in figure 6, almost overlap, thus asserting consistency in sample manufacturing and polymerization time. The obtained values for Young's modulus and tensile strength, presented in table 3, are consistent with the literature.

The density of the two constituents was determined by sample weighing and measuring the sample displaced volume (table 4).

Table 1

EXPERIMENTAL DATA FOR THE TWO LAYERED FIBREGLASSPOLYESTER RESIN COMPOSITE

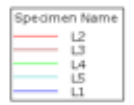

\begin{tabular}{|c|c|c|}
\hline Specimen & $\mathbf{E}[\mathbf{M P a}]$ & $\boldsymbol{\sigma}_{\mathbf{t}} \mathbf{( M P a )}$ \\
\hline $\mathrm{S} 1$ & $1.54 \mathrm{E}+04$ & 265.15 \\
\hline $\mathrm{S} 2$ & $1.52 \mathrm{E}+04$ & 253.31 \\
\hline $\mathrm{S} 3$ & $1.50 \mathrm{E}+04$ & 227.86 \\
\hline $\mathrm{S} 4$ & $1.51 \mathrm{E}+04$ & 233.15 \\
\hline S5 & $1.53 \mathrm{E}+04$ & 240.21 \\
\hline Medium value & $\mathbf{1 . 5 2 E}+04$ & $\mathbf{2 4 3 . 2 1}$ \\
\hline
\end{tabular}




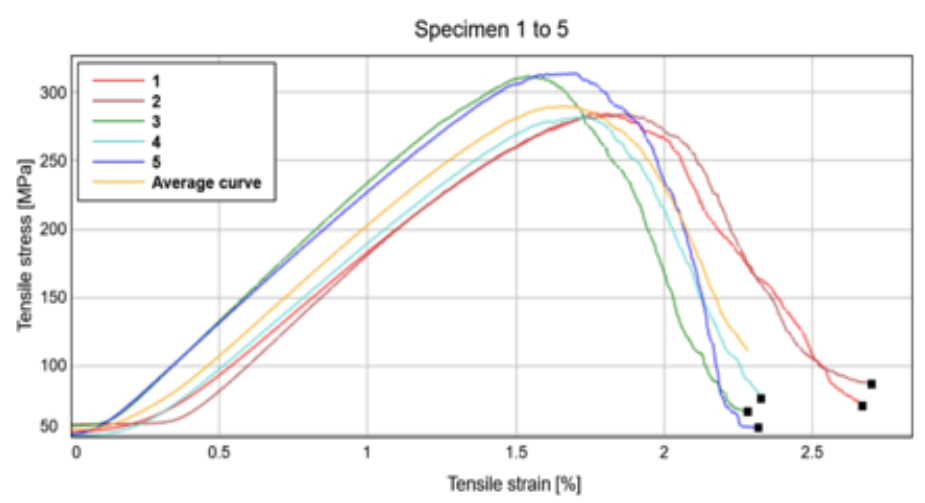

Table 2

EXPERIMENTAL RESULTS OBTAINED FOR FIBERGLASS YARN

\begin{tabular}{|c|c|c|}
\hline Specimen & E [MPa] & $\sigma_{\mathrm{t}}(\mathbf{M P a})$ \\
\hline $\mathrm{S} 1$ & $1.81 \mathrm{E}+04$ & 284.89 \\
\hline $\mathrm{S} 2$ & $1.99 \mathrm{E}+04$ & 284.45 \\
\hline $\mathrm{S} 3$ & $1.98 \mathrm{E}+04$ & 312.66 \\
\hline $\mathrm{S} 4$ & $1.84 \mathrm{E}+04$ & 282.50 \\
\hline S5 & $1.88 \mathrm{E}+04$ & 314.23 \\
\hline Mean value & $\mathbf{1 . 9 0 E}+04$ & $\mathbf{2 9 5 . 7 4 6}$ \\
\hline
\end{tabular}

Table 3

Fig. 5. Characteristic curves obtained for fiberglass yarn Specimen 1 to 5

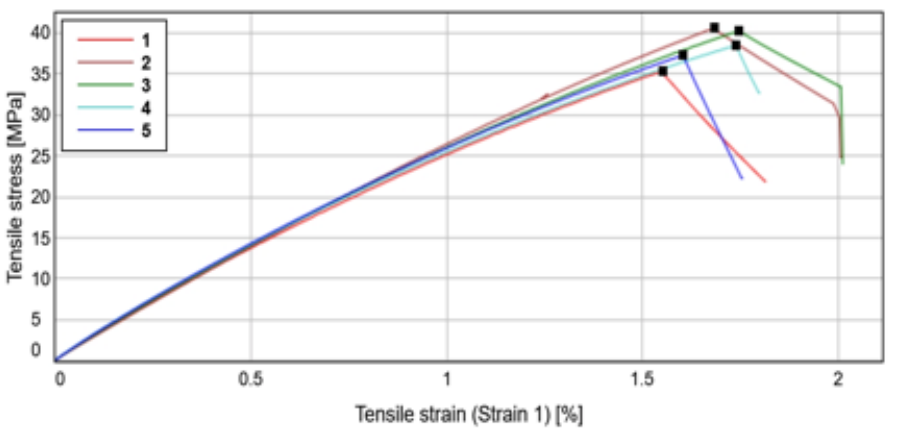

Fig. 6. Characteristic curves obtained for polyester resin

\section{Finite element modelling part}

After detailed measurement of the fibreglass yarn, woven pattern and the double-layered specimen dimensions, a meso-scale a 3D model was created using SolidWorks CAD software, with details at constituent level. The entire 3D model was created as solid bodies without the use of surface or beam approximations. In figure 7, the $3 \mathrm{D}$ model of the specimen was presented.

Considering the repeatability of component pattern and part positioning for a computer-generated 3D model, only a small fraction from the whole was sufficient for our proposed analysis. Also, in the following image (fig. 8), two symmetry planes were identified, thus allowing a more detailed mesh without overloading the computational machine. In figure 9.a a detail on the resin component was presented and figure 9.b shows a detail of the fibreglass component.

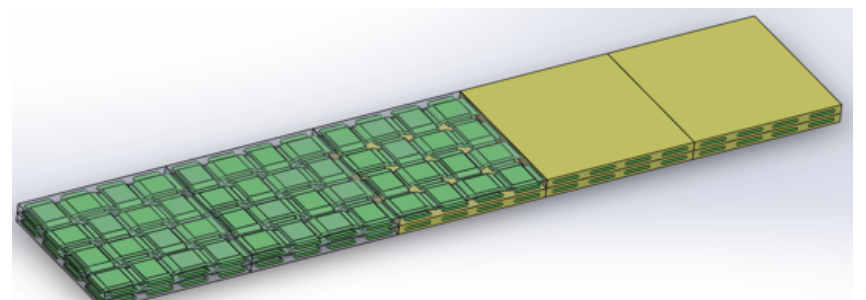

Fig. 7. SolidWorks generated 3D model with partial transparency

In order to create and solve the finite element model, the $3 \mathrm{D}$ generated specimen was imported in Ansys Workbench v.19, using the Transient Structural setup.

Considering the premises of this analysis the boundary conditions (fixed support and symmetry) were applied as is shown in figure 10. Thus, $A$ and $\mathbf{B}$ represent the symmetry plane boundary condition where $A$ locks the displacement on $X$ axis and $B$ locks the displacement on $Y$ axis. $C$ represents the fixed support.

A total displacement of $0.1 \mathrm{~mm}$ was imposed on the $Z$ axis on the yellow marked area presented in figure 11. Considering the transient structural analysis setup, the displacement was applied in ten $0.01 \mathrm{~mm}$ steps, thus

EXPERIMENTAL RESULTS OBTAINED FOR POLYESTER RESIN

\begin{tabular}{|c|c|c|}
\hline Specimen & E [MPa] & $\sigma_{\mathbf{t}}(\mathbf{M P a})$ \\
\hline P1 & 2907 & 35.4 \\
\hline P2 & 2835 & 40.6 \\
\hline P3 & 2951 & 40.3 \\
\hline P4 & 3061 & 38.5 \\
\hline P5 & 3068 & 37.3 \\
\hline Medium value & $\mathbf{2 9 6 4}$ & $\mathbf{3 8 . 4}$ \\
\hline
\end{tabular}

Table 4

THE DENSITIES OF CONSTITUENTS

\begin{tabular}{|c|c|}
\hline Constituent & Density $\left[\mathrm{kg} / \mathrm{m}^{3}\right.$ ] \\
\hline Fiberglass & 2448.2 \\
\hline Polyester resin & 1384 \\
\hline
\end{tabular}

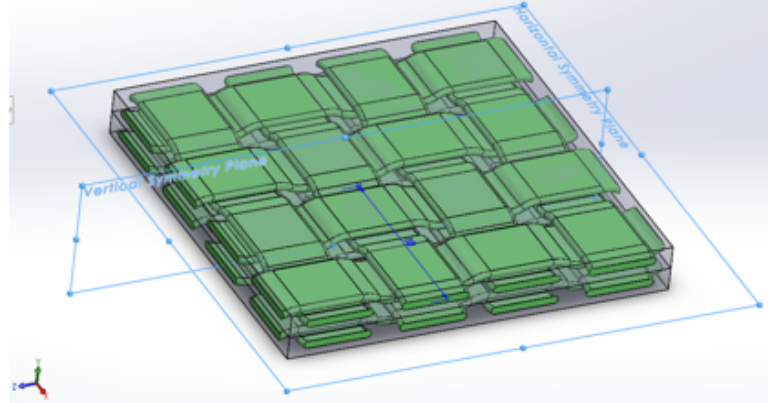

Fig. 8. Proposed 3D model for numerical analysis

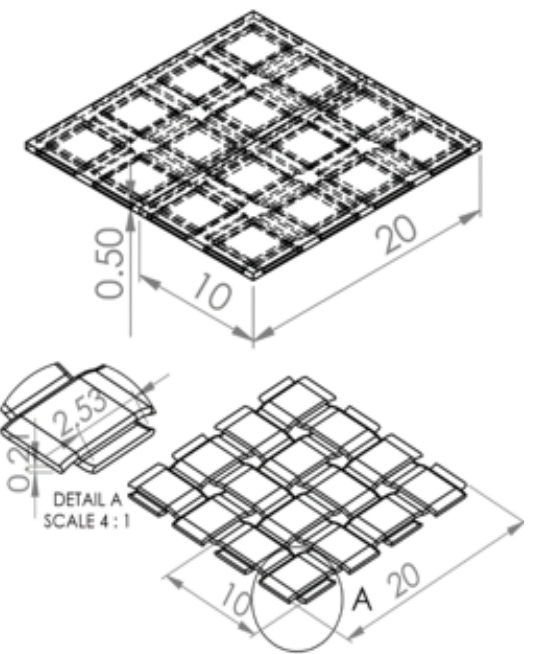

a. Resin component of the $3 \mathrm{D}$ model

b. Fibreglass component of the 3D model

Fig. 9. Consitituent level details of 3D model obtaining an evolution of the reaction force from the fixed support.

A successful mesh, on the simplified by symmetry model, was obtained using 10 node tetrahedron elements with an average size of $0.1 \mathrm{~mm}$. A total number of 414757 nodes and 220401 elements were obtained as presented in figure 12. Higher mesh density was achieved in proximity of yarn top and bottom (not depicted) side. 


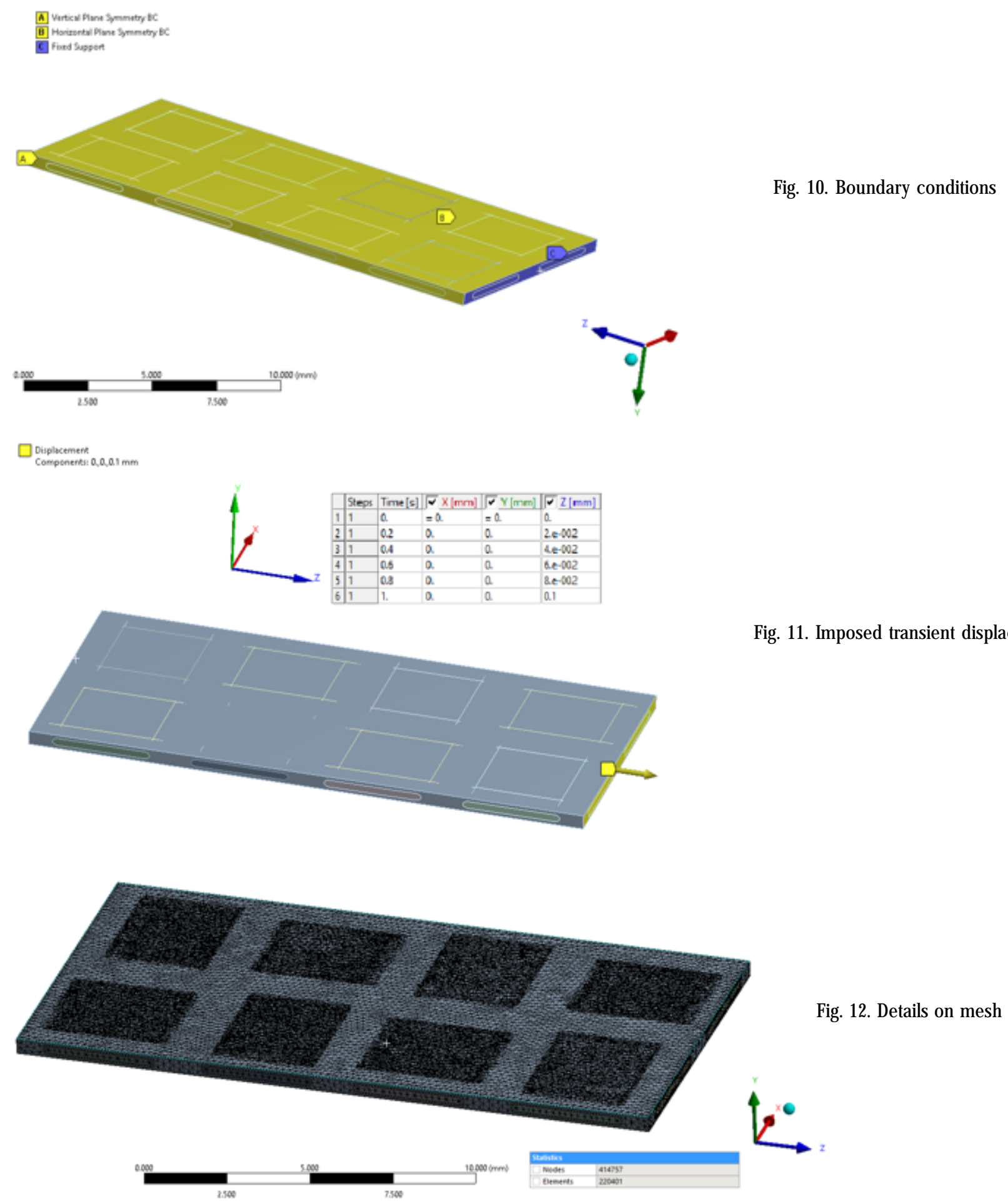

\section{Results and disscutions}

After a successful solve of the finite element model, a first set of data was extracted from the results. In figure 13 , the reaction force from the fixed support is presented alongside the force values for each step along the global axes. The non-zero values of force from $X$ and $Y$ axes were caused by the woven pattern and position inside the polyester matrix. Our interest was focused on the $Z$ axis force reaction values. These data were used in order to determine the tensile stress, for each timestep.

The second set of data to serve our interest was the normal elastic strain on Z axis located on the YZsymmetry plane of the model section. Figure 14 presents the averaged values of strain variation on model section. The side graph presents strain variation over time steps, for maximum, average and minimum values. Higher strain values were obtained in the matrix and lower values for the fibreglass yarns, corresponding to the different rigidities of the constituents.

The obtained data from FEM were summed up in table 5 alongside the calculated results on each timestep for normal Z axis stress (obtained by dividing the reaction force by the $5 \mathrm{~mm}^{2}$ cross-section area) and Young's modulus. Considering the linear elastic domain of the analysis, the proportionality between stress (reaction force) and strain was maintained resulting in a constant Young's modulus throughout timesteps.

Figure 15 presents the Strain-Stress curve for the virtual composite specimen. The proportionality relation between stress and strain highlighted by the chart. The slope calculation of the chart represents the Young's modulus.

Comparing the numerical model result for Young's modulus (1.29E +04 MPa) with the same experimental result $(1.52 \mathrm{E}+04 \mathrm{MPa})$ generates a difference of $15.13 \%$. 


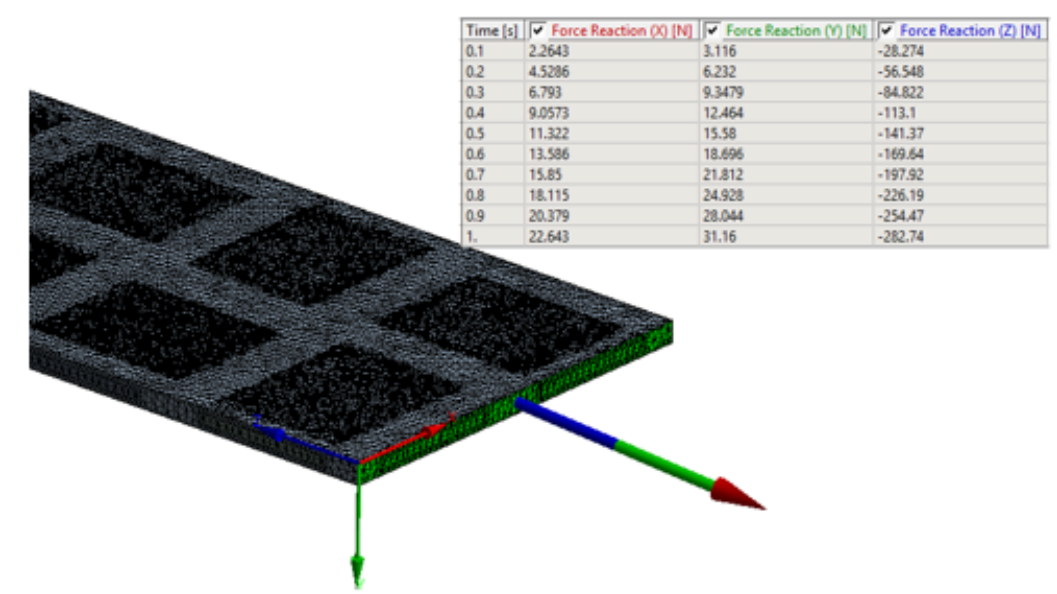

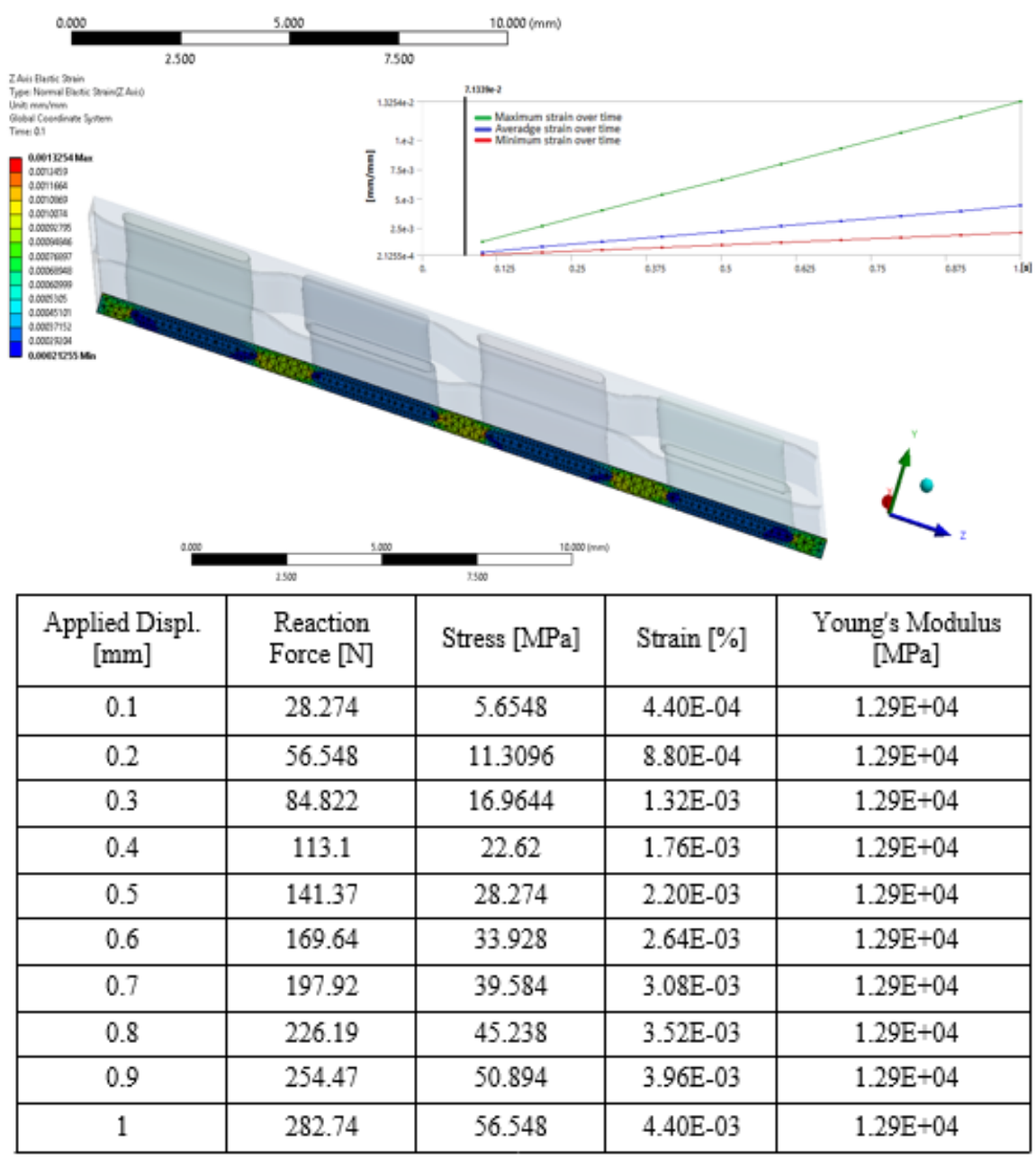

Numerical Strain-Stress Curve

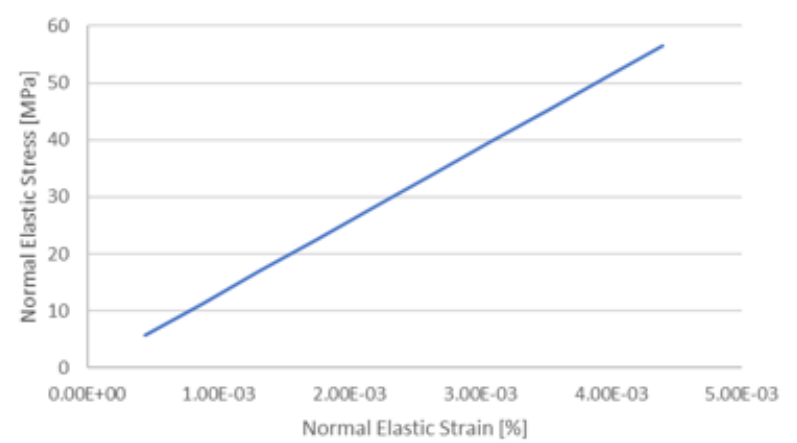

Fig. 15. Numerically obtained Strain-Stress curve

A difference value below $20 \%$ is considered acceptable in engineering. It can be considered that the finite element model was validated with room for further improvement.
Fig. 13. Reaction force

Fig. 14. Normal $Z$ axis strain variation in model symmetry YZ plane

Table 5

NUMERICAL RESULTS FOR FINITE ELEMENTS METHOD 
parameterized so that the volume ratio of fibreglass fabric/ polyester resin varies according to the designer's needs.

Other advantage consists in the possibility of extrapolating the method to any kind of fabric and resin and could be subject of further research.

Future investigations shall seek to create a model capable of predicting all the elastic characteristics required in engineering design, starting from the elastic characteristics of the constituents. It is also a goal to validate the model using the numerical method of calculation with finite differences as schematized in [15].

A possible application for this method could be in the area of vehicle safety and crashworthiness as is presented in the work [16] of Jiga G. et al.

Acknowledgements: The authors are grateful for all members from the INOVABIOMED project, ID: P_36_611:145/26.10.2016, http:// www.inovabiomed.upb.ro/ and the University POLITEHNICA of Bucharest for technical support The authors would like to thank editors and anonymous reviews. This work has been funded by University Politehnica of Bucharest, through the "Excellence Research Grants" Program, UPB-GEX 2017, Identifier: UPB-GEX-2017, Project acronim MCV.

\section{References}

1. SUN, B., LIU, R., GU, B., Numerical simulation of three-point bending fatigue of four-step 3-D braided rectangular composite under different stress levels from unit-cell approach, Computational Materials Science, 65, 2012, pp. 239-246

2. LI, D.S., FANG, D.N., JIANG, N., XUEFENG, Y., Finite element modeling of mechanical properties of $3 \mathrm{D}$ five-directional rectangular braided composites, Composites Part B: Engineering, 42(6), 2011, pp. 1373-1385

3. WU, L., ZHANG, F., SUN, B., GU, B., Finite element analyses on three-point low-cyclic bending fatigue of 3-D braided composite materials at microstructure level, International J ournal of Mechanical Sciences, 84, 2014, pp. 41-53

4. FAGIANO, C., GENET, M., BARANGER, E., LADEVĖZE, P., Computational geometrical and mechanical modeling of woven ceramic composites at the mesoscale, Composite Structures, 112, 2014, pp. 146-156

5. JACQUES, S., DE BAERE, I., VAN PAEPEGEM, W., Application of periodic boundary conditions on multiple part finite element meshes for the meso-scale homogenization of textile fabric composites, Composites Science and Technology, 92, 2014, pp. 41-54

6. SADIGHI, M., HOSSEINI, S. A., Finite element simulation and experimental study on mechanical behavior of $3 D$ woven glass fiber composite sandwich panels, Composites Part B: Engineering, 55, 2013, pp. 158-166

7. SIDDIQUI, M.Z., TARIQ, F., NAZ, N., AHMED, M.F., Determination of Young's modulus of metallic and composite materials by digital image correlation, Journal of Space Technology, 1(1), 2012, pp. 32-37

8. DRAGHICI, S., PARAUSANU, I., BACIU, F., PETRESCU, H.A., HADAR, A., PASTRAMA, S.D., A Comparative Experimental-Numerical Analysis on the Vibration Behaviour of a Composite Satellite Subset. Mat. Plast., 53, no. 4, 2016, p. 585-589

9. SAVA, M., HADÃR, A., PARAUSANU, I., PETRESCU, H.A., BACIU, F., STANESCU M.M., Validation of the numerical model of single-layer composites reinforced with carbon fiber and aramid, In AIP Conference Proceedings, Vol. 1738, No. 1, 2016, 480080

10. LI, J., JIAO, Y., SUN, Y., WEI, L., Experimental investigation of cutedge effect on mechanical properties of three-dimensional braided composites, Materials \& design, 28(9), 2007, pp. 2417-2424

11. VERPOEST, I., LOMOV, S.V., Virtual textile composites software WiseTex: Integration with micro-mechanical, permeability and structural analysis, Composites Science and Technology, 65(15-16), 2005, pp. 2563-2574

12. KABELKA, J., Prediction of the Thermal Properties of Fibre-Resin Composites. (Retroactive Coverage), Elsevier Applied Science Publishers, Ltd., Developments in Reinforced Plastics-III, 1984, pp. 167-202

13. WHITCOMB, J., WOO, K., GUNDAPANENI, S., Macro finite element for analysis of textile composites. Journal of Composite Materials, 28(7), 1994, pp. 607-618

14. MARREY, R.V., SANKAR, B.V., A micromechanical model for textile composite plates. J ournal of Composite Materials, 31(12), 1997, pp. 1187-1213

15. DUNCA, G., IOVANEL, R.G., BUCUR, D.M., CERVANTES, M.J., On the Use of the Water Hammer Equations with Time DependentFriction during a Valve Closure, for Discharge Estimation, Journal of Applied Fluid Mechanics, 9(5), 2016

16. JIGA, G., STAMIN, a ., DINU, G., VLASCEANU, D., POPOVICI, D., Material and shape crash-box influence on the evaluation of the impact energy absorption capacity during a vehicle collision, Ciencia \& Tecnologia dos Materiais, 28(1), 2016, pp. 67-72

Manuscript received:28.07.2018 